\title{
A Query Unit for the IPSec Databases
}

Alberto Ferrante,

Satish Chandra

$$
\text { ALaRI, }
$$

University of Lugano

E-mail: \{ferrante, kaverips\}@alari.ch

\author{
Vincenzo Piuri \\ DTI, \\ University of Milano \\ E-mail: piuri@dti.unimi.it
}




\section{Outline}

IPSec

The Database Query $\underline{\text { Unit }}$

Multithreaded Unit

Simulations

Conclusions and

Future Work

\section{IPSec}

\section{The Database Query Unit}

Multithreaded Unit

Simulations

Conclusions and Future Work 


\section{IPSec}

\section{IPSec}

\section{IPSec}

$\mathrm{AH}, \mathrm{ESP}$

Databases

Security

Associations

Main IPSec

Processing Steps

Database Query

The Database Query Unit

Multithreaded Unit

Simulations

Conclusions and

Future Work $\checkmark$ Is a suite of protocols

$\boldsymbol{x}$ adding security at IP (network) level;

$\checkmark$ makes extensive use of cryptographic functions. 


\section{AH, ESP}

IPSec

IPSec

$\mathrm{AH}, \mathrm{ESP}$

Databases

Security

Associations

Main IPSec

Processing Steps

Database Query

The Database Query Unit

Multithreaded Unit

Simulations

Conclusions and

Future Work $\checkmark \quad$ IPSec is mainly composed of two protocols:

x Authentication Header $(\mathrm{AH})$;

x Encapsulating Security Payload (ESP);

$\checkmark$ both protocols can be used in:

$x$ transport mode;

$\boldsymbol{x}$ tunnel mode. 


\section{Databases}

\section{IPSec}

\section{IPSec}

$\mathrm{AH}, \mathrm{ESP}$

\section{Databases}

Security

Associations

Main IPSec

Processing Steps

Database Query

The Database Query Unit

Multithreaded Unit

Simulations

Conclusions and

Future Work
IPSec uses two databases:

$\boldsymbol{x}$ the Security Policy Database (SPD);

$\boldsymbol{x}$ the Security Association Database (SAD):

$\checkmark$ the records are the Security Associations (SAs). 


\section{Security Associations}

\section{$\underline{\text { IPSec }}$}

IPSec

$\mathrm{AH}, \mathrm{ESP}$

Databases

Security

Associations

Main IPSec

Processing Steps

Database Query

The Database Query Unit

Multithreaded Unit

Simulations

Conclusions and

Future Work $\checkmark$ Each SA contains:

$x$ protocol/algorithms settings;

$\boldsymbol{x}$ keys for cryptographic algorithms;

$\checkmark$ SAs are mono-directional:

$x$ two SAs need to be created for normal bidirectional communications. 


\section{Main IPSec Processing Steps}

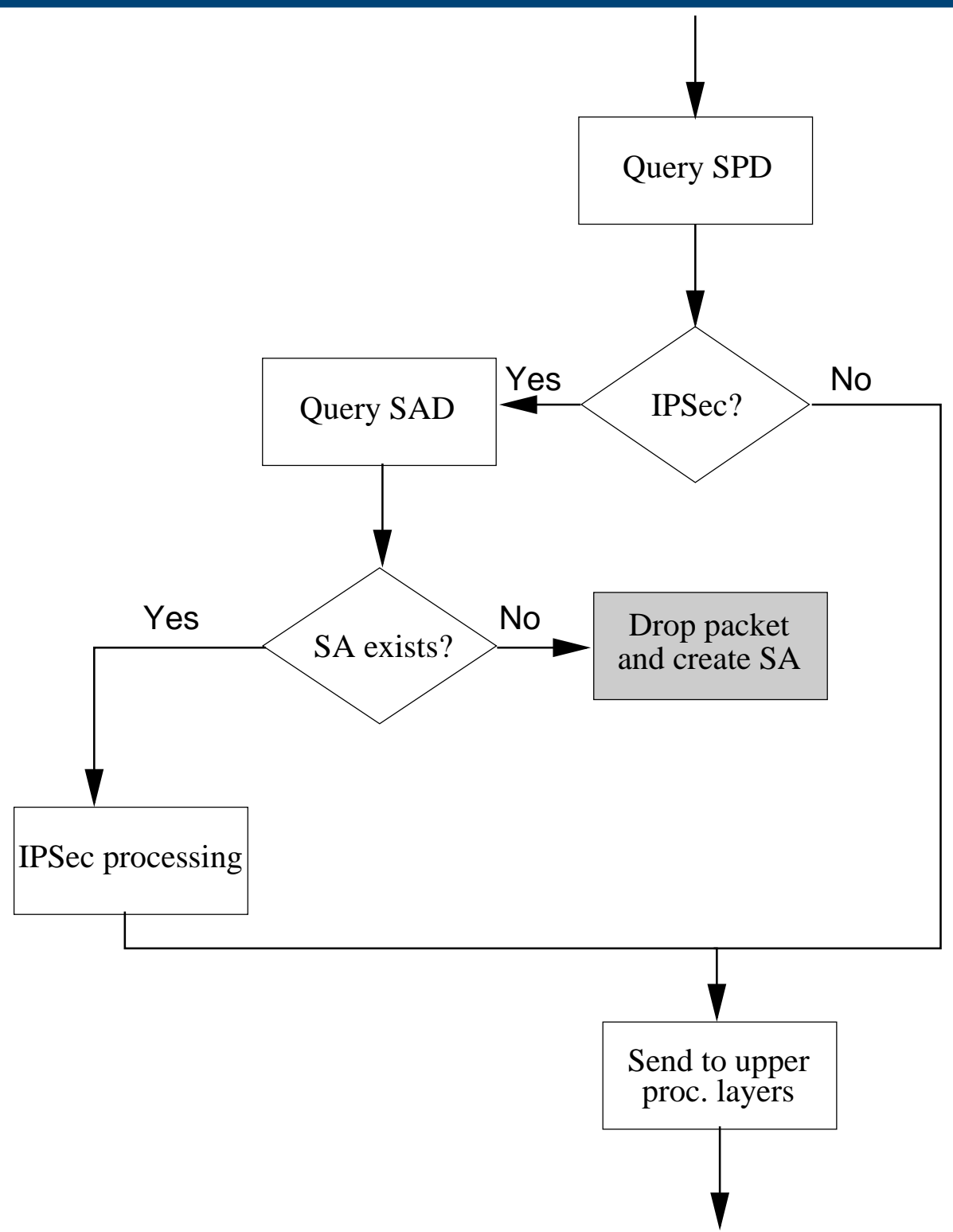




\section{Database Query}

$\underline{\mathrm{IPSec}}$

IPSec

$\mathrm{AH}, \mathrm{ESP}$

Databases

Security

Associations

Main IPSec

Processing Steps

Database Query

The Database Query Unit

Multithreaded Unit

Simulations

Conclusions and

Future Work $\checkmark$ More than 3 million queries/s in a $1 \mathrm{Gbit} / \mathrm{s}$ system (worst case);

$\boldsymbol{\sim}$ may be a bottleneck;

$\checkmark$ may become a weak point (DoS). 


\section{High-level Architecture}

IPSec

The Database Query Unit

High-level

Architecture

How it Works

Record Size

Memory Query

Techniques

Cache Replacement

Policies

Multithreaded Unit

\section{Simulations}

Conclusions and

Future Work

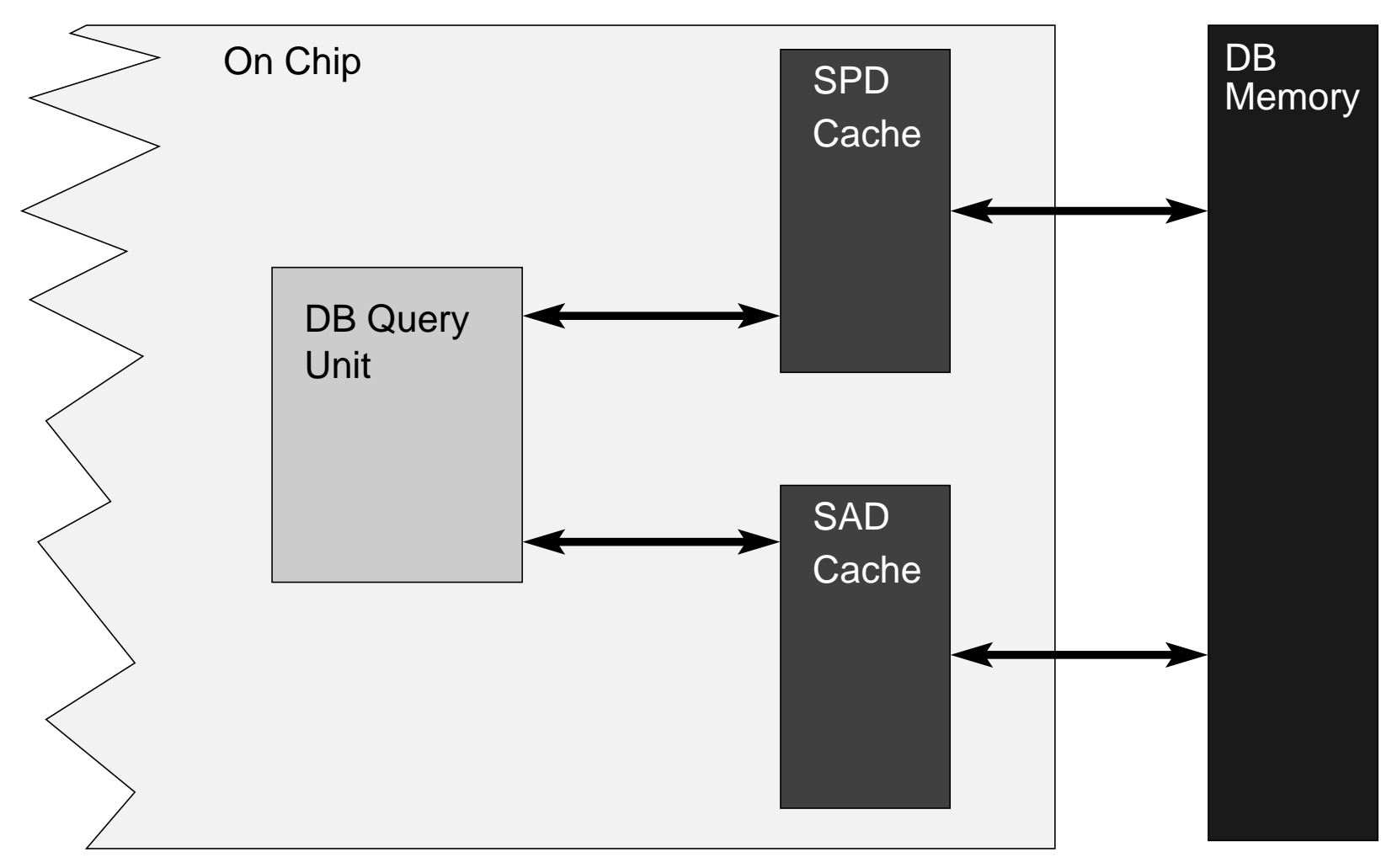

Caches are Content Addressable Memories. 


\section{How it Works}

IPSec

The Database Query Unit

High-level

Architecture

How it Works

Record Size

Memory Query

Techniques

Cache Replacement

Policies

Multithreaded Unit

Simulations

Conclusions and

Future Work $\checkmark$ SPD query:

$\boldsymbol{x}$ cache query;

$\boldsymbol{x}$ main DB query if not in cache;

$\checkmark$ SAD query:

$x$ cache query;

$x$ main DB query if not in cache:

$\checkmark$ SPD-provided pointer. 


\section{Record Size}

\section{IPSec}

The Database Query

\section{Unit}

High-level

Architecture

How it Works

\section{Record Size}

Memory Query

Techniques

Cache Replacement

Policies

Multithreaded Unit

Simulations

Conclusions and

Future Work
SPD:

$\checkmark$ two parts:

$\boldsymbol{X}$ repeatedly used information (IP, SA pointers, ... );

$\boldsymbol{X}$ rarely used information (proposals);

$\checkmark$ repeatedly used information (232 bits) are cached.

SAD:

all fields are repeatedly used (792 bits). 


\section{Memory Query Techniques}

\section{IPSec}

The Database Query Unit

High-level

Architecture

How it Works

Record Size

Memory Query

Techniques

Cache Replacement

Policies

Multithreaded Unit

Simulations

Conclusions and

Future Work $\boldsymbol{\checkmark}$ Linear LookUp Technique (LLUT);

$x$ memory queried in a linear fashion;

$\checkmark$ Partitioned LookUp Technique (PLUT).

$x$ memory divided into pages;

$x$ IP address is used

to associate a record to a page;

$\boldsymbol{x}$ linear search inside the pages;

x "fragmentation" problem. 


\section{Cache Replacement Policies}

IPSec

The Database Query Unit

High-level Architecture

How it Works

Record Size

Memory Query

Techniques

Cache Replacement

Policies

Multithreaded Unit

Simulations

Conclusions and

Future Work

\author{
First In First Out; \\ Least Recently Used.
}




\section{Parallelizing Queries}

$\underline{\text { IPSec }}$

The Database Query $\underline{\text { Unit }}$

Multithreaded Unit Parallelizing Queries

Simulations

Conclusions and Future Work
Queries in memory take a long time; other queries in cache can be done during this time; parallel queries related to the same SA are not allowed. 


\section{Simulation Description}

IPSec

The Database Query $\underline{\text { Unit }}$

Multithreaded Unit

Simulations

Simulation

Description

Design Space

Queries/second

Sequential System:

SAD and SPD

Query Times

Multithreaded

System: SAD and

SPD Query Times

Conclusions and

Future Work $\checkmark$ SystemC functional model;

$\boldsymbol{v}$ simulates behavior and delays

of the blocks;

$\boldsymbol{V}$ input: ITA tracefiles. 


\section{Design Space}

\section{IPSec}

The Database Query Unit

Multithreaded Unit

Simulations

Simulation

Description

\section{Design Space}

Queries/second

Sequential System:

SAD and SPD

Query Times

Multithreaded

System: SAD and

SPD Query Times

Conclusions and

Future Work $\checkmark 168$ different configurations;

$\checkmark$ LLUT for the first 84 configurations, PLUT for the others;

$\checkmark$ FIFO for odd configurations, LRU for even ones.

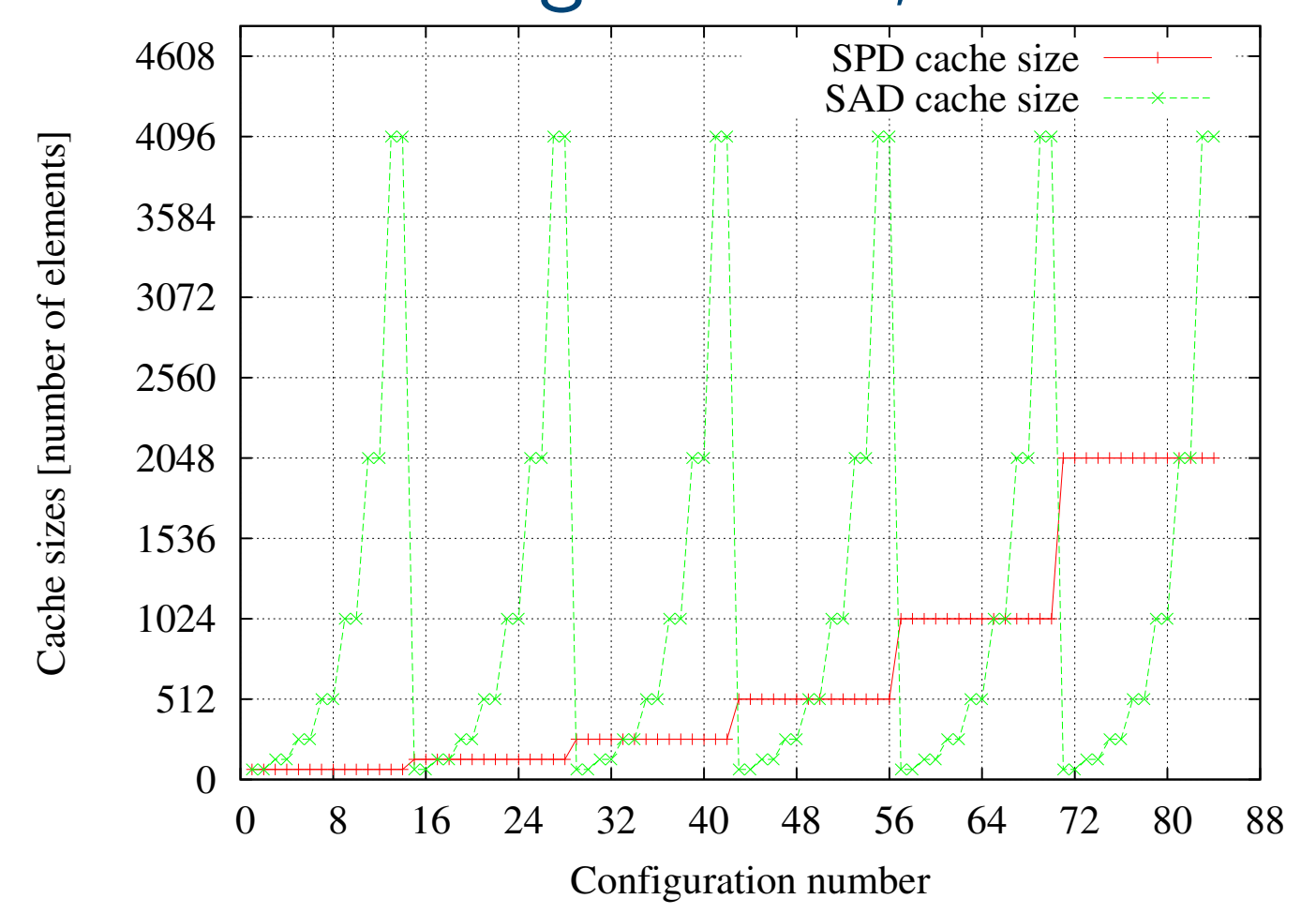




\section{Queries/second}
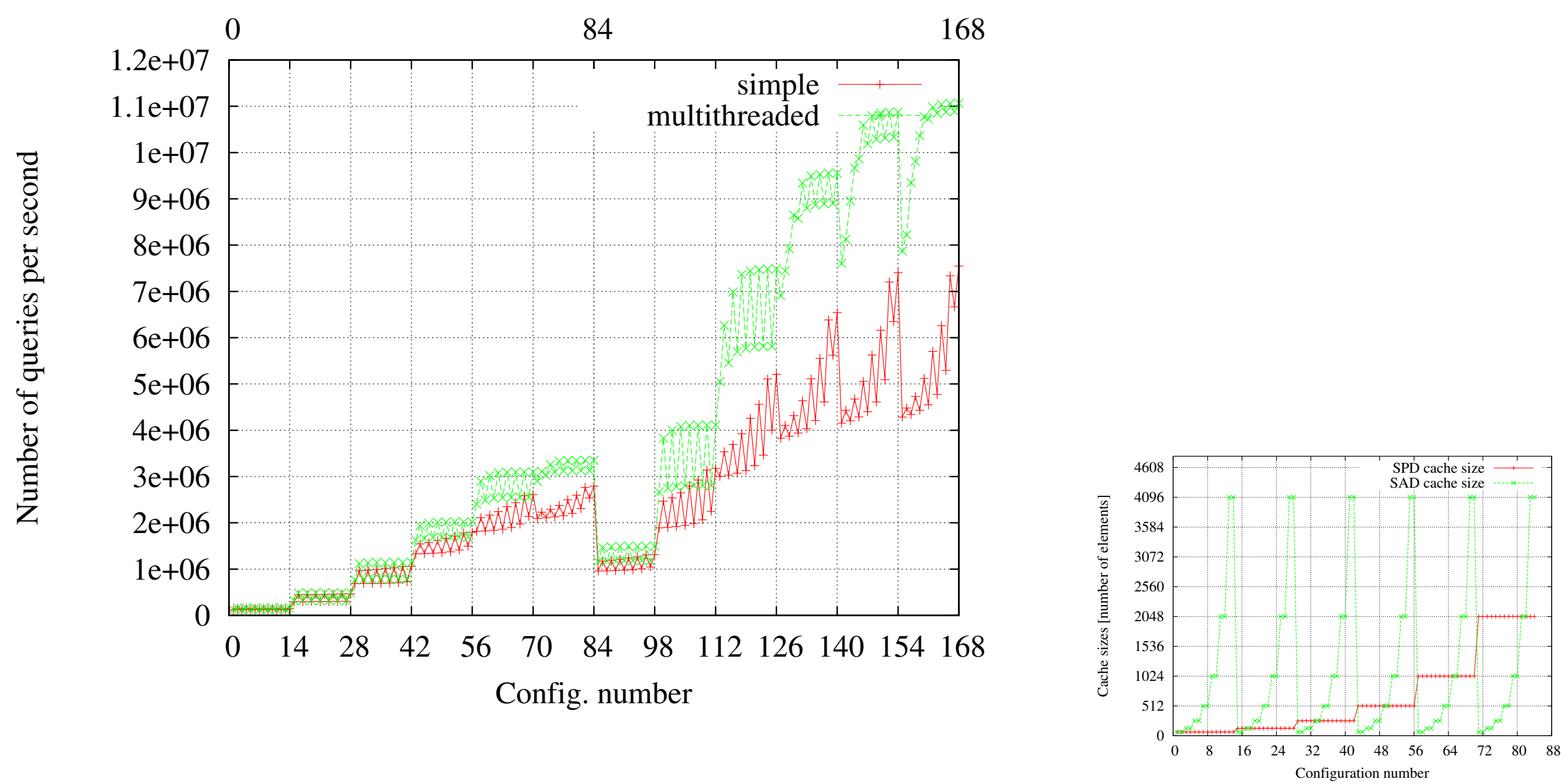

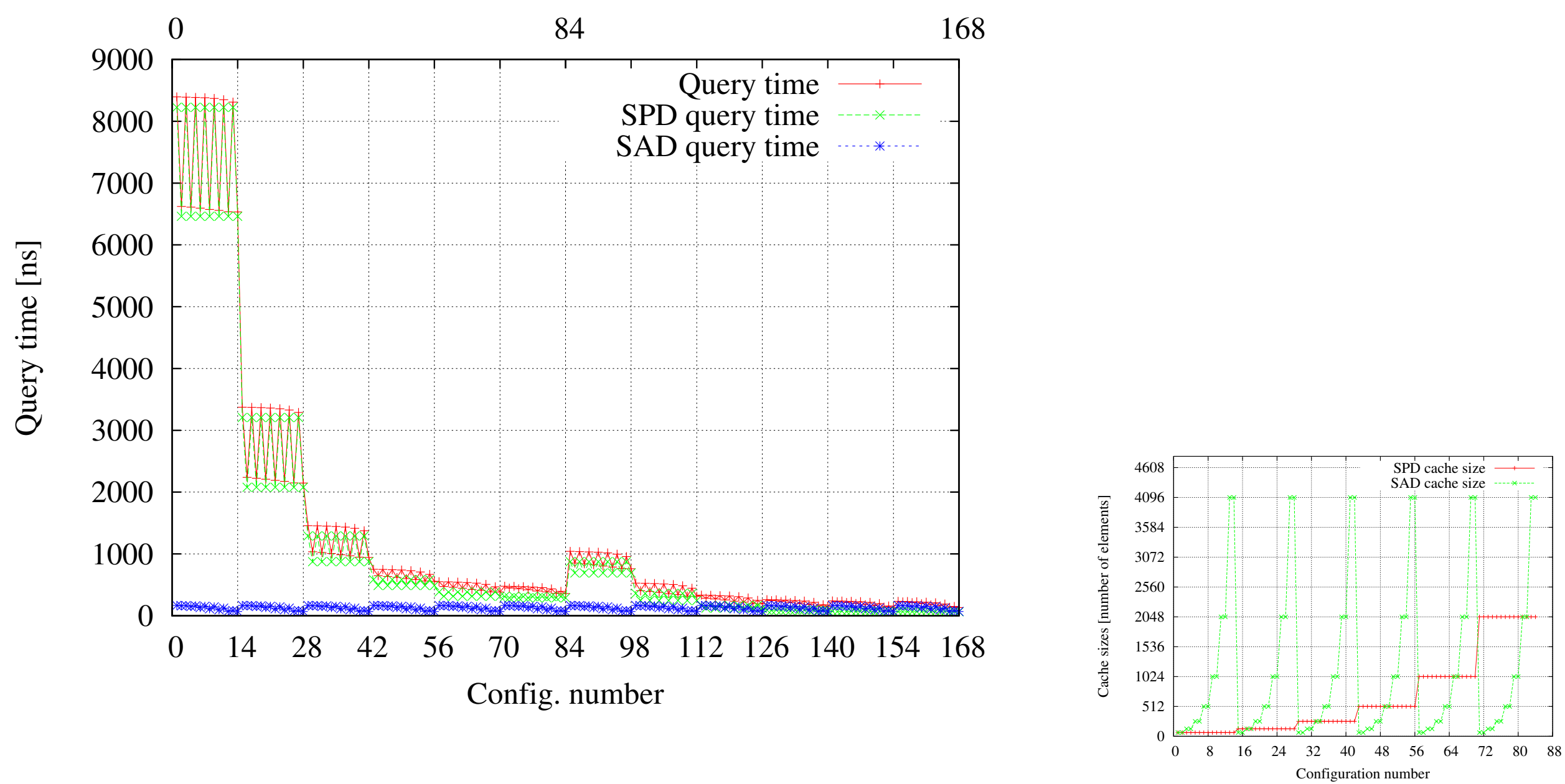

Query Times
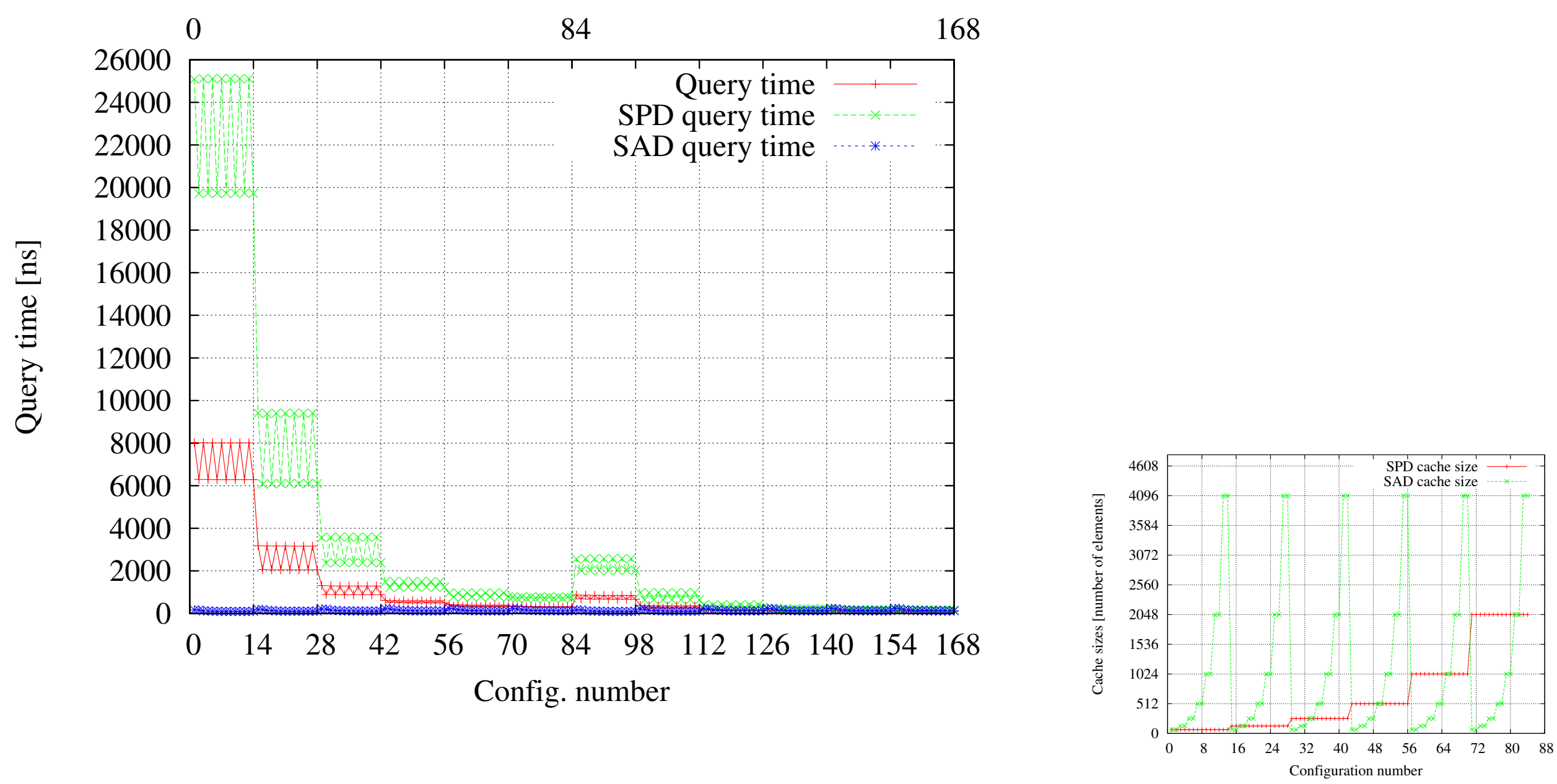


\section{Conclusions and Future Work}

\section{IPSec}

The Database Query $\underline{\text { Unit }}$

Multithreaded Unit

Simulations

Conclusions and

Future Work

Conclusions and Future Work
We designed a DB query unit:

$\checkmark$ able to exceed

11 million queries per second;

$\checkmark$ efficient.

Future Work:

$\checkmark$ more accurate simulations;

$\checkmark$ out of order queries. 